\title{
Expression of Rho Kinase and Its Mechanism in the Left Atrial Appendage in Patients with Atrial Fibrillation
}

\author{
Chen Yongqing, ${ }^{*}$ Su Fangju, ${ }^{*}$ Han Juanping, Jiao Piqi, Guo Wenyun \\ Department of Cardiology, Lanzhou General Hospital, Lanzhou, China
}

\section{ABSTRACT}

Aim: To study the expression of Rho kinase (Rho associated coil forming protein kinase-1, ROCK-1) and its substrate myosin phosphatase target subunit 1 (myosin phosphatase target subunit-1, MYPT-1), connexin $40(\mathrm{Cx} 40)$ and connexin $43(\mathrm{Cx} 43)$ in the left atrial appendage of patients with atrial fibrillation, and explore the role of ROCK signaling pathway in patients with atrial fibrillation and its underlying mechanism.

Methods: 40 patients undergoing open heart surgery were divided into two groups; atrial fibrillation group ( $\mathrm{AF}$ group) and sinus rhythm group (SR group). About $100 \mathrm{mg}$ of left atrial appendage tissue was taken during surgery and quickly frozen in liquid nitrogen. Immunohistochemistry and western blot were performed to evaluate the expression and location of ROCK-1, MYPT-1, Cx40 and Cx43 in the left atrial appendage tissue.

Results: The results indicated that the expression of ROCK-1, MYPT-1, and Cx40 in the left atrial appendage in patients with atrial fibrillation was significantly upregulated $(P<.01)$, the difference in the two groups was statistically significant, and ROCK-1, Cx40, and MYPT-1 expression in the $\mathrm{AF}$ group were higher than those in sinus rhythm group; there was a weakly positive expression of $\mathrm{Cx} 43$ protein in the $\mathrm{AF}$ group and sinus rhythm group, the difference was not statistically significant, and ROCK-1 and MYPT- 1 expression showed a significant positive correlation $(\mathrm{r}=0.968$, $P<.05)$, MYPT 1 and Cx40 protein expression was also positively correlated $(\mathrm{r}=0.983, \mathrm{P}<.05)$. Evidence in the left atrial appendage tissue of patients with atrial fibrillation showed that some proteins in Rho/ROCK pathway were upregulated, and MYPT-1 and Cx40 protein expression in $\mathrm{AF}$ group were significantly higher than that of SR group, which was also positively correlated; $\mathrm{Cx} 43$ showed a weak positive expression in both the SR group and AF group, which indicates that Rho kinase may induce expression of Cx40 by phosphorylation of MYPT-1; Cx43 may not be

Received June 10, 2017, 2017; received in revised form August 8, 2017; August 12, 2017.

*Both authors contributed equally to this work.

Funding: Natural Science Foundation of Gansu Province (No. 1308R7ZA135)

Correspondence: Prof. Yongqing Chen, MD, Department of Cardiology, Lanzhou General Hospital, 333 Southern Binhe Road, Lanzhou 730050, China; +86931-8994225 (e-mail:nemobyx@126.com). involved, suggesting that Rho kinase signaling pathway may activate and play an important role in the pathogenesis of atrial fibrillation lesions.

\section{INTRODUCTION}

Atrial fibrillation $(\mathrm{AF})$ is a common arrhythmia with high disability and mortality rate. The risk of cardiovascular diseases in patients with atrial fibrillation is significantly higher. As a member of the small G protein superfamily, Rho exerts GTPase activity. Rho/Rho kinase signal pathway is a common pathway in the human body. Rho kinase, also known as Rho associated coil forming protein kinase (ROCK), is the downstream effector molecule of Rho. Rho kinase comprises two subtypes: ROCK-1 and ROCK-2. There is a high expression of ROCK-1 in heart, lung, skeletal muscles, kidneys, and pancreas [Higashi 2003]. Myosine phosphatae targeting subunit 1 (MYPT-1) in myosin light chain phosphatase (MLCP) is the primary substrate of activated ROCK [Kimura 1996]. Rho kinase can inhibit MLCP by phosphorylation of MYPT-1 [Ito 2004]. Therefore, high phosphorylation level of MYPT-1 may reflect the activation of Rho/ROCK pathway. Many studies have shown that increased Rho kinase activity often leads to dysfunction of the cardiovascular system [Zhou 2011]. Connexin remodeling plays an important role in the incidence and development of atrial fibrillation [Pellman 2010]. Rho/Rho kinase signaling pathway is a hot topic in research concerning cardiovascular diseases; however, its relation to the pathogenesis of atrial fibrillation remains unknown.

In this study, the functional mechanism of Rho kinase was preliminarily explored through two steps: first, the expression level of ROCK- 1 and the change of activated MYPT-1 were evaluated in tissue samples taken from $\mathrm{AF}$ and $\mathrm{SR}$ group, which could clarify the role of the Rho/Rock signal transduction pathway in the pathogenesis of AF. Second, the relationship between MYPT-1 and Cx proteins in the left atrial tissues in patients with $\mathrm{AF}$ were analyzed, and the mechanism underlying the activation of Rho kinase in $\mathrm{AF}$ was explored.

\section{MATERIALS AND METHODS}

An immunohistochemistry kit was purchased from Wuhan Boster Co. MYPT1, ROCK-1, Cx40 and Cx43 antibody, and GAPDH were all purchased from Santa Cruz (USA), and prestained protein molecular weight marker was purchased from Fermentas (Lithuania). 


\section{Patient Data}

A total of 40 patients (12 males, 28 females) 20 to 68 years of age (mean age: 44) underwent thoracic surgery (mainly for rheumatic heart disease, congenital heart disease, atrial septal defect and ventricular septal defect and so on) in the Lanzhou military region general hospital during 2014. According to atrial fibrillation morbidity, the patients were divided into two groups: sinus rhythm group consisting of 20 cases and atrial fibrillation group consisting of 20 cases (6 paroxysmal atrial fibrillation and 14 persistent atrial fibrillation). Criteria for the diagnosis of atrial fibrillation was defined by the replacement of $\mathrm{P}$ wave by a series of tiny, differently shaped $f$ waves of irregular frequency in an electrocardiogram test and the appearance of pulse deficit, arrhythmia, and cardiac tone of irregular force in the physical examination. Paroxysmal atrial fibrillation, which has a usual duration of less than 48 hours, can be spontaneously converted to sinus rhythm within seven days, while persistent atrial fibrillation often lasts for more than seven days and can be converted to sinus rhythm with medication. In addition to routine preoperative electrocardiogram and echocardiography, coronary arteriography should be employed for patient over 50 to rule out coronary heart disease. The preoperative heart function of all patients was classified as I-II according to the New York Heart Association (NYHA) functional classification system. There was no significant difference in sex composition ratio, age, and heart function between the two groups. Left atrial appendage tissue samples weighing about $100 \mathrm{mg}$ each were cut from each patient's heart and immediately put on ice and stored at $-80^{\circ} \mathrm{C}$ within 20 min of the cardiac operation. All tissue specimens were obtained with the approval of the ethics committee of the Lanzhou military region general hospital.

\section{Immunohistochemistry}

Expression of MYPT-1, ROCK-1, Cx40, and Cx43 in atrial tissue was detected by IHC. In brief, tissues in paraffin sections were treated with the following procedures: dewaxing, incubation with first and secondary antibody, DAB staining, and mounting with anti-fade oil. PBS was used as a negative control. Positive slides supported by Wuhan Boster (China) were taken as positive control. The dilution of primary antibody and secondary antibody were 1:50, 1:500 respectively. Cellular location analysis of ROCK- 1 and MYPT-1 was observed under a microscope after immunohistochemical staining, and protein expression of $\mathrm{Cx} 40$ and Cx43 was calculated by Image-Pro Plus 6.0 software and presented as the mean optical density value. Differences and comparison of the two protein expressions in the left atrial appendage tissue were performed by GraphPad Prism (Version 6).

\section{Western Blot}

Expression of MYPT-1, ROCK-1, and $\mathrm{Cx} 43$ was analyzed by western blot. In brief, atrial muscle tissues were precooled in liquid nitrogen; then lysate buffer RIPA was added to it and grinded into homogenizing tissues. After centrifugation, the supernatant was collected and aliquoted. Using $100 \mu \mathrm{g}$ protein and mixing with $5 \times$ protein loading buffer, the sample mixture was boiled for $5 \mathrm{~min}$ and centrifuged before loading. After adding protein marker, electrophoresis was performed under constant voltage of $80 \mathrm{~V}$ for $1.5 \mathrm{~h}$ to $2 \mathrm{~h}$. Next, protein transfer to NC membrane was accomplished under constant voltage of $20 \mathrm{~V}$ for $1.5 \mathrm{~h}$. After separation from PAGE gel, NC membrane was blocked in 5\% non-fat milk (dissolved in TBS-T) at room temperature for $1.5 \mathrm{~h}$ with slow shaking. The first antibody including MYPT-1, ROCK-1, and Cx43 was diluted by $5 \%$ milk/PBST at 1:300 ratio and incubated overnight at $4^{\circ} \mathrm{C}$. After TBS-T washing three times, the secondary antibody of goat-anti-rabbit was diluted by $5 \%$ milk/ TBST at 1:2000 ratio and incubated at room temperature for $1 \mathrm{~h}$. Again, the membrane was washed three times by TBS-T (15 min each time). The membrane was put in enhanced chemiluminescence (ECL) substrate for $2 \mathrm{~min}$ and the film was pressed and developed before detection of protein expression. Gray scale and area of the protein band were analyzed by a gel imaging system and protein expression level was indicated by the integral gray value (D, density).
A

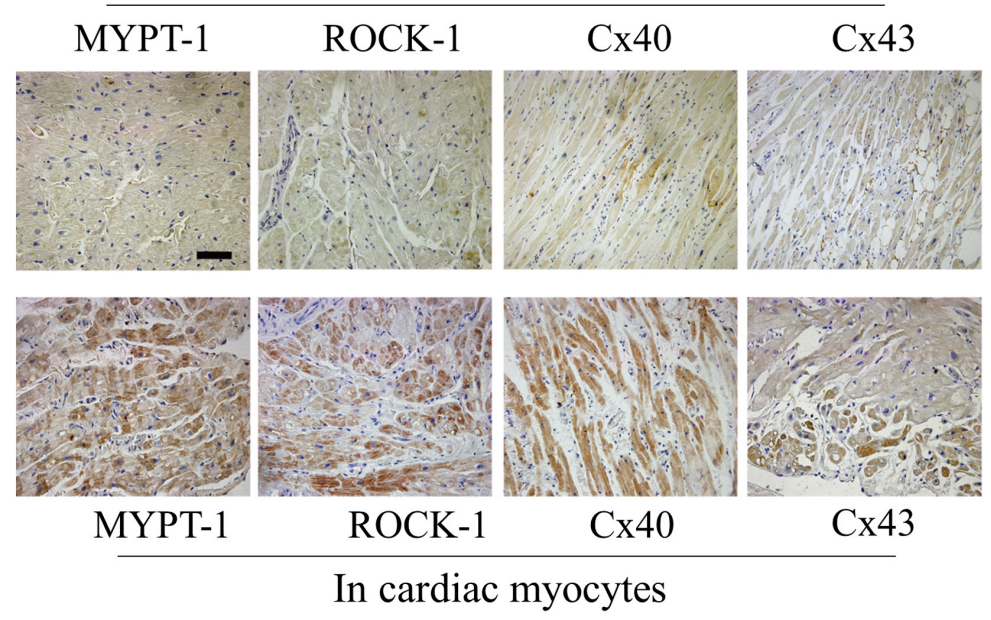

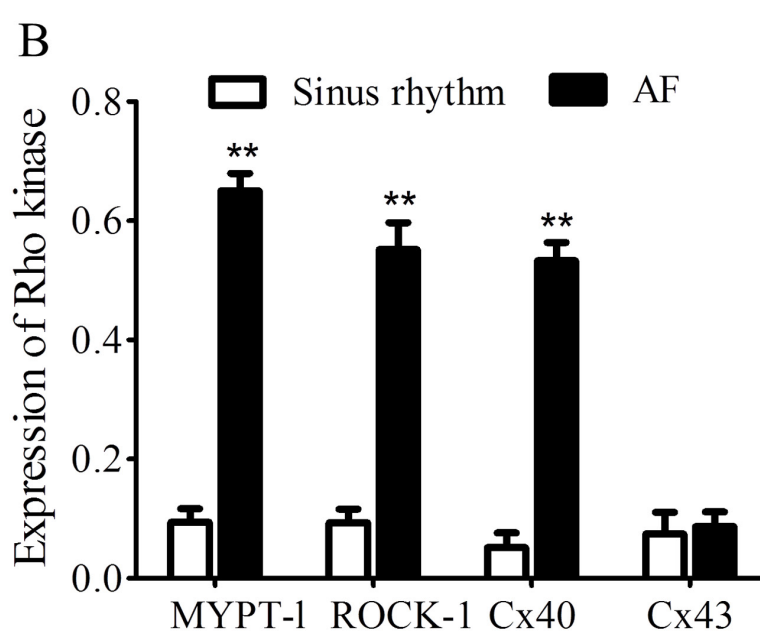

Figure 1. The immunohistochemical staining results in different myocardial cells groups. A, upper panel, the expression of MYPT-1, ROCK-1, Cx40, Cx43 is present as brown-yellow spots in sinus myocardium cell respectively; lower panel, the expression of MYPT-1, ROCK-1, Cx40, Cx43 is shown as brown-yellow spots in cardiac myocytes, which also indicated that the above proteins are mainly distributed in cytoplasm. Scale bar $=50 \mu \mathrm{m}$. B, the quantification of the brown-yellow spots of different proteins using Imagepro-Plus. $* * P<.01$, compared with sinus rhythm group. 

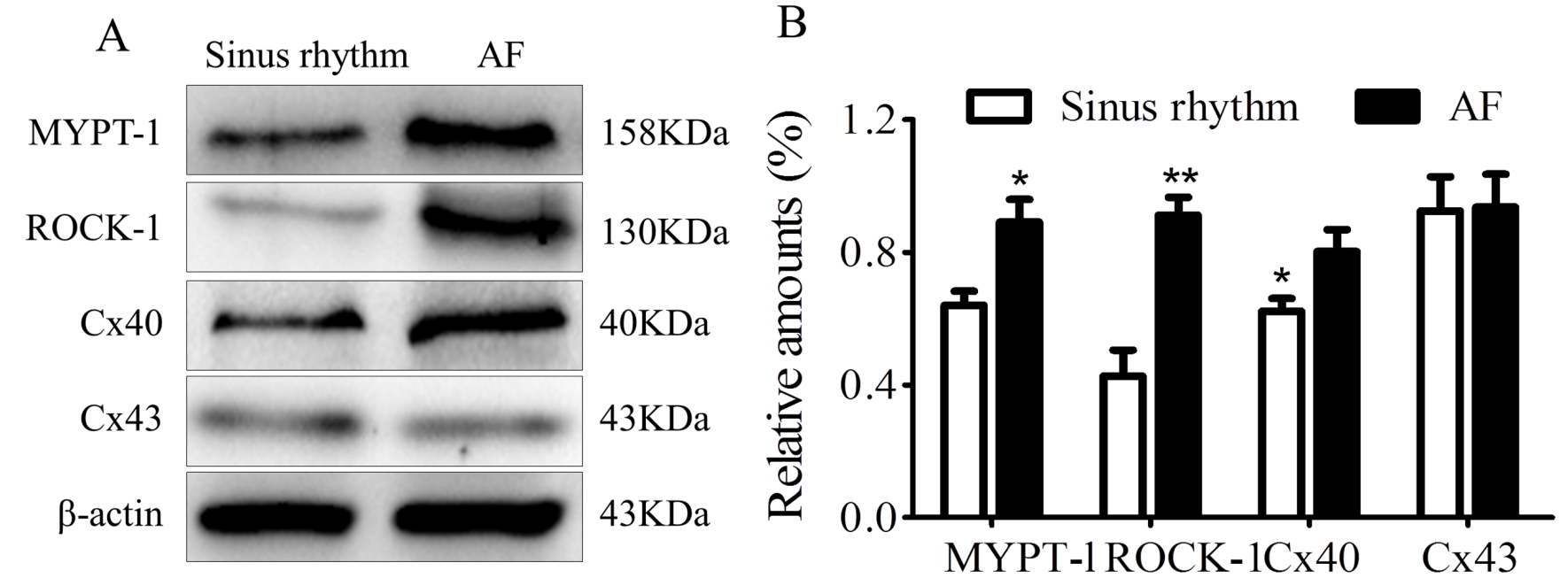

Figure 2. Western Blot results of ROCK-1, MYPT-1, Cx40, and Cx43 in the two groups. A, the original pictures. B, the quantification of the gray scale of the bands in differently treated groups by software Image J. $* P<.05, * * P<.01$, compared with sinus rhythm group.

\section{Statistical Analysis}

All statistical analyses were performed using the SPSS 17.0 statistical software package. The ANOVA test was employed to evaluate differences in the expression of protein between two categories of tissues. Pearson Spearman test was used to analyze the relationship between MYPT- 1 and $\mathrm{Cx} 40 . P<.05$ in all cases was considered statistically significant.

\section{RESULTS}

\section{Expression of MYPT-, ROCK-1, Cx40 and Cx43 in Left Atrial Appendage}

Cells positive for MYPT-1, ROCK-1, Cx40, and Cx43 were stained yellow or brown in the cytoplasm, and those unstained indicated the negative expression of the above markers. All of the MYPT-1, ROCK-1, Cx40, and Cx43 were mildly expressed in the left auricle (or left atrial appendage) of sinus rhythm (SR group), as observed under a microscope. A high expression of MYPT-1, ROCK-1, and Cx40 was observed in that of the atrial fibrillation group (AF group), while $\mathrm{Cx} 43$ was mildly expressed (Figure 1).

\section{Protein Levels of ROCK-1, MYPT-1, Cx40 and Cx43 in Different Groups}

As shown in Figure 2, the expression of ROCK-1, MYPT-1, $\mathrm{Cx} 40$, and $\mathrm{Cx} 43$ was inconsistent with the immunochemistry results above; the expression of MYPT-1, ROCK-1, and Cx40 were significantly higher in the $\mathrm{AF}$ group than those of the $\mathrm{SR}$ group.

\section{Correlation Analysis}

As shown in Figure 3, the expression of ROCK-1 protein showed positive correlation with the expression of MYPT-1 protein after the relative quantification of immunohistochemistry $(\mathrm{r}=0.968, P<.05) .(\mathrm{r}=0.983, P<.05)$. The expression of ROCK-1 protein was positively correlated with the expression of $\mathrm{Cx} 40$ protein $(\mathrm{r}=0.963, P<.05)$.

\section{DISCUSSION}

Rho family is a kind of small G proteins with GTP enzyme activity, which in recent years was found to be widely expressed in all eukaryotic kingdoms. Their functions include molecular switch, swift between GTP-binding state (active) and GDP-binding state (inactive), triggering downstream kinase cascade reaction that results in various biological responses [Wettschureck 2002; Ishizaki 1997; Narumiya 1996]. There are three isoforms of Rho (A, B, and C) out of which Rho A is of paramount importance. Its downstream protein Rho kinase (ROCK) has been proven to play a pivotal role in cardiac functions. ROCK with molecular weight of $160 \mathrm{kD}$ belongs to threonine/serine protein kinase and comprises three domains; $\mathrm{N}$-terminal kinase domain, coil domain with Rho binding site in the middle, and $\mathrm{C}$-terminal cysteine-rich $\mathrm{PH}$ domain [Noma 2006; Loirand 2006]. ROCK1 and ROCK2 are highly homologous isoforms with $65 \%$ similarity in their amino acid sequences. Their similarity ratio can reach up to $92 \%$ in their kinase domain. ROCK1 is widely expressed in heart, lungs, skeletal muscles, kidneys, and pancreas [Higashi 2003].

Experimental studies have shown a close association between Rho/ROCK pathway and a variety of cardiovascular diseases. For example, data collected from experimental studies on both animal [Kandabashi 2000] and human models [Kandabashi 2002] show a significantly increased expression of ROCK mRNA expression in atherosclerotic lesions; rat models of experimental hypertension including both spontaneously hypertensive rats and chronic NO synthesis inhibition-induced hypertensive rats showed increased Rho/ROCK activity [Uehata 1997]. In a model of pulmonary hypertension induced by monocrotaline, ROCK inhibitor Fasudil improved pulmonary vascular remodeling and relieved the contraction of smooth muscle cells, thereby reducing pulmonary hypertension [Chapados 2006]. It also indirectly indicates the involvement of Rho/ROCK activation in the formation of pulmonary hypertension. Our results showed that there is a higher expression of ROCK-1 and MYPT-1 in auricula sinistra in the atrial fibrillation group than in the sinus rhythm group, which suggests that the activation of Rho/ROCK pathway is involved in the incidence of atrial fibrillation. It has been found that at least 25 different substrates of ROCK exist, most of which are involved in actin filament formation, and the organization and rearrangement of cytoskeleton [Shimokawa 2005]. Among them, myosin 
A

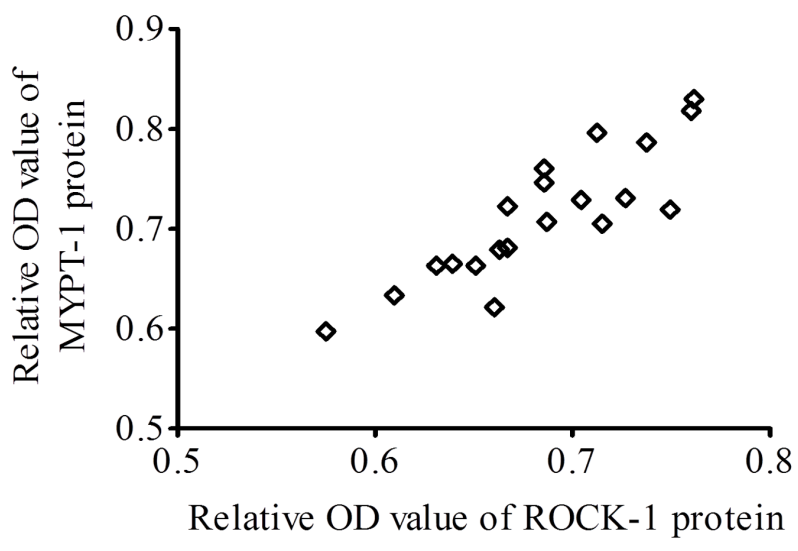

B

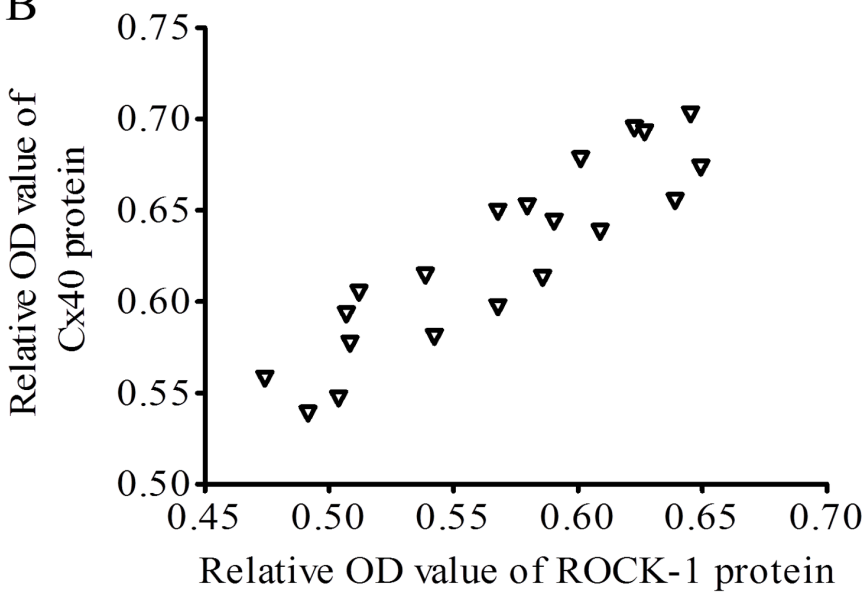

Figure 3. The correlation analysis of protein expression between MYPT-1 and ROCK-1 or Cx40 and ROCK-1. A, relative OD value of MYPT-1 protein and ROCK-1 protein. $\mathrm{B}$, relative OD value of $\mathrm{C} \times 40$ protein and ROCK-1 protein.

light chain phosphatase (MLCP) is the most studied protein [Mam 2010; Nossaman 2010], which consists of one catalytic domain, a myosin light chain binding subunit (MYPT-1), and the noncatalytic structural subunit. ROCK can interact with the MYPT-1 of MLCP [Furuyama 2006]. Therefore, in this study, we first detected the expression of one subtype of Rhokinase, ROCK-1 and MYPT-1 in the left atrial appendage of atrial fibrillation patients, which may indicate the activation of Rho pathway.

Numerous studies have confirmed that ROCK is involved in oxidative stress, inflammation, fibrosis, apoptosis, ventricular remodeling and other biological processes by targeting its downstream molecules such as MYPT-1, cardiac troponin, PENT, MAPK, and their pathways. Atrial fibrosis and gap junction connexin $(\mathrm{Cx})$ remodeling plays an essential role in AF progression [Pellman 2010; Severs 2004], so an important question arises here: what does the $\mathrm{Cx}$ have to do with Rho kinase pathway? As an important component of the intercalated discs of myocardium, the gap junction (GJ) is the tunnel that transmits key electrical and chemical signals between myocardial cells, which in turn participates in the coordination of action potential and myocardial cell movement synchronization [Rackauskas 2010; Jansen 2010]. Connexin 40 and Connexin 43 are two important members of gap junction proteins in the human heart [Dupays 2003; Wang 2010]. Connexin 40 is mainly expressed in human atrial muscle, atrioventricular node, His bundle, left and right bundle branch, and proximal Purkinje fibers [Dupays 2003].

Mitogen-activated protein kinase (MAPK) is a serine/threonine protein kinase within the cells. In mammals there are three parallel MAPK pathways; extracellular signal-regulated kinase (ERK) pathway, c-jun N-terminal kinase (c-JNK) pathway, and p38 MAPK pathway. MAPK signaling networks are found in ventricular and atrial muscles of the heart. Evidence has shown that RhoA/ROCK pathway plays a regulatory role in these three MAPK signaling pathways [Wang 2010; Shatanawi 2011]. Studies have proven that the activation of ERK pathway (major and classical pathway in the MAPK pathway family) in cardiac cells results in various biological effects, such as proliferation of cardiac fibroblasts, increased matrix collagen and connexin remodeling through a series of complex signaling network.
Therefore, our future study will focus on the expression of $\mathrm{Cx} 40 / \mathrm{Cx} 43$ and their correlation with MYPT- 1 in the left atrial appendage (LAA) of patients with atrial fibrillation (AF). Our previous studies suggested that the expression of $\mathrm{Cx} 40$ in the LAA of AF patients is significantly higher than those in the sinus rhythm group, which might be closely related to the imbalanced synthesis and degradation of connexin and the different sites of serine/threonine phosphorylation or dephosphorylation. However, in our study, the expression of Cx43 in LAA didn't show significant difference between AF patients and SR group. This is consistent with the study of Polontchouket al, who observed that $\mathrm{Cx} 40$ is 2.7 times more highly expressed in LAA of AF patients than in SR group, while $\mathrm{Cx} 43$ is expressed in the same degree in both $\mathrm{AF}$ patients and SR group.

Our results show that patients with atrial fibrillation show significantly higher expression of MYPT-1 and Cx40 in the left atrial appendage tissue than those in the sinus rhythm group, suggesting that activation of Rho/ROCK pathway is involved in the occurrence of atrial fibrillation. We also found expression of Cx40 MYPT-1 positively correlated in the AF group. In summary, our study indicates that Rho/ROCK may activate MYPT-1, thus upregulating the expression of $\mathrm{Cx} 40$, which in turn participates in the mechanism that leads to atrial fibrillation, a detailed molecular mechanism that needs to be further studied.

\section{REFERENCES}

Chapados R, Abe K, Ihida-Stansbury K, et al. 2006. ROCK controls matrix synthesis in vascular smooth muscle cells: coupling vasoconstriction to vascular remodeling. Circ Res 99:837-44.

Dupays L, Mazurais D, Rücker-Martin C, et al. 2003. Genomic organization and alternative transcripts of the human Connexin 40 gene. Gene 305:79-90.

Furuyama T, Komori K, Shimokawa H, et al. 2006. Long-term inhibition of Rho kinase suppresses intimal thickening in autologous vein grafts in rabbits. J Vasc Surg 43:1249-56.

Higashi M, Shimokawa H, Hattori T, et al. 2003. Long-term inhibition of Rho-kinase suppresses angiotensin II-induced cardiovascular hypertrophy in rats in vivo: effect on endothelial $\mathrm{NAD}(\mathrm{P}) \mathrm{H}$ oxidase system. Circ Res 93:767-75. 
Ishizaki T, Naito M, Fujisawa K, et al. 1997. p160ROCK, a Rho-associated coiled-coil forming protein kinase, works downstream of Rho and induces focal adhesions. FEBS Lett 404:118-24.

Ito K, Hirooka Y, Kishi T, et al. 2004. Rho/Rho-kinase pathway in the brainstem contributes to hypertension caused by chronic nitric oxide synthase inhibition. Hypertension 43:156-62.

Jansen JA, van Veen TA, de Bakker JM, et al. 2010. Cardiac connexins and impulse propagation. J Mol Cell Cardiol 48:76-82.

Kandabashi T. 2000. Inhibition of myosin phosphatase by upregulated rho-kinase plays a key role for coronary artery spasm in a porcine model with interleukin-1beta. Circulation 101:1319-23.

Kandabashi T, Shimokawa H, Mukai Y, et al. 2002. Involvement of rhokinase in agonists-induced contractions of arteriosclerotic human arteries. Arterioscler Thromb Vasc Biol 22:243-8.

Kimura K, Ito M, Amano M, et al. 1996. Regulation of myosin phosphatase by Rho and Rho-associated kinase (Rho-kinase). Science 273:245-8.

Loirand G, Guerin P, Pacaud P. 2006. Rho kinases in cardiovascular physiology and pathophysiology. Circ Res 98:322-34.

Mam V, Tanbe AF, Vitali SH, et al. 2010. Impaired vasoconstriction and nitric oxide-mediated relaxation in pulmonary arteries of hypoxia- and monocrotaline-induced pulmonary hypertensive rats. J Pharmacol Exp Ther 332:455-6.

Narumiya S. 1996. The small GTPase Rho: cellular functions and signal transduction. J Biochem 120:215-28.

Noma K, Oyama N, Liao JK. 2006. Physiological role of ROCKs in the cardiovascular system. Am J Physiol Cell Physiol 290:C661-8.

Nossaman BD, Nossaman VE, Murthy SN, et al. 2010. Role of the
RhoA/Rho-kinase pathway in the regulation of pulmonary vasoconstrictor function. Can J Physiol Pharmacol 88:1-8.

Pellman J, Lyon RC, Sheikh F. 2010. Extracellular matrix remodeling in atrial fibrosis: mechanisms and implications in atrial fibrillation. J Mol Cell Cardiol 48:461-7.

Rackauskas M, Neverauskas V, Skeberdis VA. 2010. Diversity and properties of connexin gap junction channels. Medicina (Kaunas) 46:1-12.

Severs NJ, Coppen SR, Dupont E, et al. 2004. Gap junction alterations in human cardiac disease. Cardiovasc Res 62:368-77.

Shatanawi A, Romero MJ, Iddings JA, et al. 2011. Angiotensin II-induced vascular endothelial dysfunction through RhoA/Rho kinase/p38 mitogen-activated protein kinase/arginase pathway. Am J Physiol Cell Physiol 300: C1181-92.

Shimokawa H, Takeshita A. 2005. Rho-kinase is an important therapeutic target in cardiovascular medicine. Arterioscler Thromb Vasc Biol 25:1767-75.

Uehata M, Ishizaki T, Satoh H, et al. 1997. Calcium sensitization of smooth muscle mediated by a Rho-associated protein kinase in hypertension. Nature 389:990-4.

Wang N, Guan P, Zhang JP, et al. 2010. Fasudil hydrochloride hydrate, a Rho-kinase inhibitor, suppresses isoproterenol-induced heart failure in rats via JNK and ERK1/2 pathways. J Cell Biochem 112:1920-9.

Wettschureck N, Offermanns S. 2002. Rho/Rho-kinase mediated signaling in physiology and pathophysiology. J Mol Med (Berl) 80:629-38.

Zhou Q, Gensch C, Liao JK. 2011. Rho-associated coiled-coil-forming kinases (ROCKs): potential targets for the treatment of atherosclerosis and vascular disease. Trends Pharmacol Sci 32:167-73. 